Check for updates

Cite this: Soft Matter, 2017,

13,4412

Received 11th January 2017

Accepted 25th May 2017

DOI: $10.1039 / \mathrm{c} 7 \mathrm{sm} 00068 \mathrm{e}$

rsc.li/soft-matter-journal

\section{Conducting microhelices from self-assembly of protein fibrils $\dagger$}

\author{
Fredrik G. Bäcklund, ${ }^{a}$ Anders Elfwing, (D)*a Chiara Musumeci, (D) ${ }^{a}$ Fátima Ajjan, ${ }^{a}$ \\ Viktoria Babenko, ${ }^{b}$ Wojciech Dzwolak, ${ }^{b}$ Niclas Solin ${ }^{a}$ and Olle Inganäs ${ }^{a}$
}

\begin{abstract}
Herein we utilize insulin to prepare amyloid based chiral helices with either right or left handed helicity. We demonstrate that the helices can be utilized as structural templates for the conducting polymer alkoxysulfonate poly(ethylenedioxythiophene) (PEDOT-S). The chirality of the helical assembly is transferred to PEDOT-S as demonstrated by polarized optical microscopy (POM) and Circular Dichroism (CD). Analysis of the helices by conductive atomic force microscopy (c-AFM) shows significant conductivity. In addition, the morphology of the template structure is stabilized by PEDOT-S. These conductive helical structures represent promising candidates in our quest for $\mathrm{THz}$ resonators.
\end{abstract}

The helical shape is a standard geometry of conductors in electromagnetics, enabling the coupling of electrical current and magnetic fields. Accordingly, such structures are utilized in a wide range of applications such as electronics and sensing; especially since fabrication of such helical geometries is straightforward at the macroscopic length scale ranging from millimeters to meters. However, at the mesoscopic scale, approaching the micro- and sub-micrometer dimension, top down fabrication of helical structures is more challenging. ${ }^{1}$ At the molecular length scale helical geometries are common, with two prominent examples being the protein alpha-helix and the DNA double helix. Furthermore, synthetic polymers can have helical conformations. ${ }^{2}$ A plethora of helical structures of mesoscopic dimensions are formed in many biological systems but it is also possible to use self-assembly in vitro, as was recently demonstrated by formation of helical microstructures via DNA origami. ${ }^{3}$ In addition, it has been

\footnotetext{
${ }^{a}$ Department of Physics, Chemistry, and Biology, Biomolecular and Organic

Electronics, Linköping University, 58183 Linköping, Sweden. E-mail: aelf@ifm.liu.se

${ }^{b}$ Department of Chemistry, Biological and Chemical Research Centre,

University of Warsaw, Pasteura 1, 02-093 Warsaw, Poland

$\dagger$ Electronic supplementary information (ESI) available: S1 Photo of PEDOTS:protein complex pellet, S2 Phototos of washing solutions, S3 Calibration curve for unbound PEDOT-S determination, S4 CD spectra of PEDOT-S decorated superstructures on glass, S5 High resolution SEM images of PEDOT-S covered superstructures. Experimental details. See DOI: 10.1039/c7sm00068e
}

demonstrated that naturally occurring structures can be isolated and act as templates for functionalization with conductors. For example, by depositing metals onto either spiral vessels of vascular plant ${ }^{4}$ or the helical microalgae Spirulina, ${ }^{5}$ conducting helical structures with resonances in the $\mathrm{THz}$ regime can be obtained. Although naturally occurring structures are abundant, their use often requires the use of cumbersome isolation and purification techniques. An alternative is the formation of helical structures by means of self-assembly of biomolecular systems in vitro. This methodology has several attractive features, such as potential tunability of product structures as well as less demanding purification steps compared to isolation from e.g. plants. Proteins have an extremely rich supramolecular chemistry, and are excellent candidates for preparation of materials by self-assembly, having dimensions in the nano- and micrometer scale. ${ }^{6}$ A prominent example is the conversion of soluble protein molecular precursors into fibrillar structures, known as amyloid fibrils, with diameters in the nanometer range and lengths in the micrometer range. ${ }^{7,8}$ Such fibrils form colloidal dispersions stabilized by electrostatic repulsion, and can be used as a scaffold for functionalization by other molecules and polymers. ${ }^{9,10}$ We have previously shown that insulin amyloid fibrils can be functionalized with conjugated polyelectrolytes, and the resulting structures, in case of conducting polymers, can be incorporated into devices such as transistors. ${ }^{11,12}$ Dzwolak and co-workers have shown that bovine insulin can form chiral helical superstructures consisting of amyloid-like fibrils. ${ }^{13-15}$ Such superstructures are formed when controlled agitation is applied during the fibril formation process. Critical for the formation and stability of helical super-structures is a well-chosen $\mathrm{pH}$ and a relatively high salt concentration (see below for further discussion of the formation of such structures). The mechanism of superstructure formation has been studied computationally by Gruziel et al. ${ }^{16}$

Herein we investigate if previously developed methodology for functionalization of amyloid fibrils with metallic conjugated polyelectrolytes can be extended to include such helical superstructures, and if chirality of the protein superstructure can be transferred to the achiral polyelectrolyte. We also investigate 
a

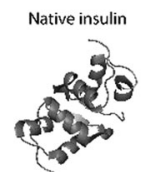

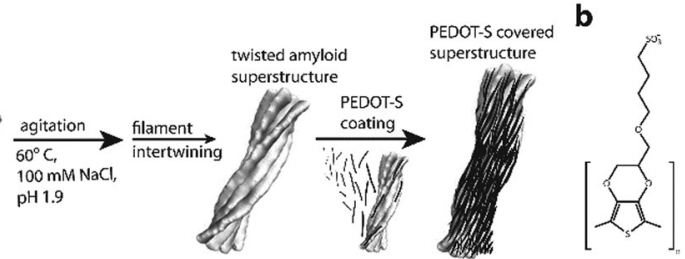

Scheme 1 (a) Schematic illustration of the preparative procedure. (b) The structure of the conducting polymer alkoxysulfonated poly(ethylenedioxythiophene), abbreviated as PEDOT-S.

the conductivity of the resulting complexes as well as how the stability of the superstructures is affected by functionalization.

PEDOT-S (the molecular structure is shown in Scheme 1b) is a relative small rod-like polyelectrolyte with an approximate length of about 16 monomers, with a conductivity of $30 \mathrm{~S} \mathrm{~cm}^{-1} .^{17}$ PEDOT-S is an electron rich conjugated polymer where, at acidic $\mathrm{pH}$, the polymer backbone readily reacts with protons. ${ }^{18}$ In the situation where the corresponding side chain sulfonate groups are deprotonated, the positive charges on the polymer backbone are balanced by negative charges on the side chain sulfonate groups. Because of this PEDOT-S can be described as a self-doped polymer. ${ }^{19}$ Generally there will be an excess of deprotonated sulfonate groups, and accordingly PEDOT-S will have a net negative charge which will enable favorable electrostatic interactions with positively charged structures. We have previously shown that PEDOT-S readily attaches to insulin amyloid fibrils at low $\mathrm{pH}$, creating electrical conductive nanowires. ${ }^{11,12}$ In a similar manner, at the $\mathrm{pH}(\mathrm{pH}$ 1.9) used for preparation of chiral superstructures of insulin, the fibril superstructures will be positively charged (the isoelectric point of insulin being approximately 5.3) and thus have a strong tendency to adhere to PEDOT-S. Using a similar approach as in previous works ${ }^{12}$ we added a large excess $(10: 1 \mathrm{w} / \mathrm{w})$ of PEDOT-S to the chiral superstructure expecting the polymer to coat the protein complex. Using centrifugation at $1000 \mathrm{~g}$, a dark blue pellet is formed, consisting of the PEDOT-S:protein complex (Fig. S1, ESI $\dagger$ ). The excess of PEDOT-S can be removed simply by washing of the resulting complex by repeated centrifugation and resuspension in MilliQ water. At the end of the process only PEDOT-S bound to the chiral superstructures remains. Since it was possible to differentiate between bound PEDOT-S (located in the pellet) and unbound PEDOT-S (located in the supernatant) it was possible to estimate the weight ratio between bound PEDOT-S and insulin fibril superstructures to be close to 1:1 (for further information about the procedure, see ESI $\dagger$ and Fig. S2 and S3). The size of the protein superstructures enables them to be readily observed by light microscopy, and we therefore turned to polarized optical microscopy (POM) to investigate their optical properties. In POM the visible light from a halogen lamp (i.e. white light) passes through a linear polarizer, a sample, and finally through a second polarizer (the analyzer). If the polarizer and analyzer are set perpendicular to each other, i.e. in a cross polarizer setting, birefringent objects will appear bright against a black background since they twist the incoming polarized light before it enters into the analyzer. Wet samples of

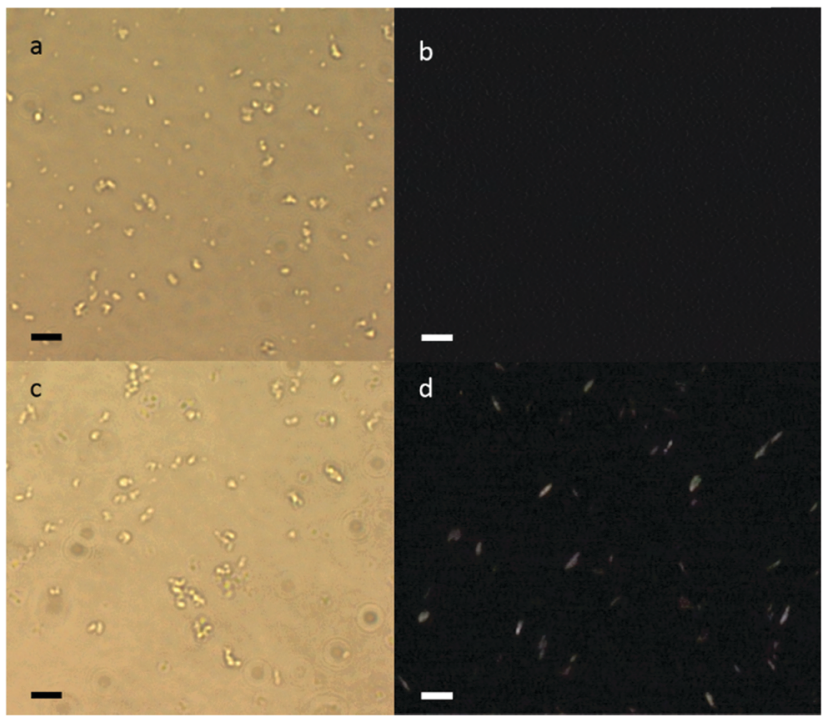

Fig. 1 Optical microscopy images of a wet film of fibril superstructures with and without PEDOT-S. The native structures are shown in phase contrast (a) and polarized microscopy (b). The corresponding images of structures with PEDOT-S are shown in phase contrast (c) polarized optical microscopy (d). The scale bar is $5 \mu \mathrm{m}$.

superstructures were studied by phase contrast microscopy as well as by POM (see Fig. 1). Phase contrast imaging revealed that the samples contained a large amount of elongated particles with approximate dimensions of 5-10 micrometer in length and 2-5 micrometer in width. Comparison between naked (Fig. 1a) and coated superstructures (Fig. 1c) showed little difference between the two types of sample. On the other hand comparison between naked and coated superstructures demonstrated that only PEDOT-S covered structures (Fig. 1d) exhibit visible birefringence; in contrast the corresponding image of naked protein superstructures (Fig. 1b) showed no birefringence.

A video recording of a wet sample of PEDOT-S covered superstructures observed by means of POM shows birefringent objects appearing and disappearing over time (ESI, $\uparrow$ Video V1). Notably, the brightness appears to vary mainly depending on the direction of the long axis of the individual objects relative to the crossed polarizers at any given moment, rather than because of objects simply drifting in and out of focus. This is as expected for an aqueous dispersion of birefringent structures undergoing random motions. In contrast, a dried sample should display fixed birefringent objects. When investigating a dried sample, it was possible to gradually change the orientation of a set of crossed polarizers relative to fixed objects of PEDOT-S functionalized superstructures and thus observing the corresponding gradual change in intensity (Fig. 2). This can be explained by the objects having a dominant linear optical axis along their long axis. If this optical axis is tilted relative to both the polarizer and the analyzer (the second polarizer in the crossed polarizer set) of the crossed polarizer set as in Fig. 2a, b and e, f, the object will transfer light to the analyzer. If, however, the optical axis is oriented near to parallel with the plane of vibration of the incoming light as in Fig. 2c and d, very little light will pass through the subsequent analyzer. 

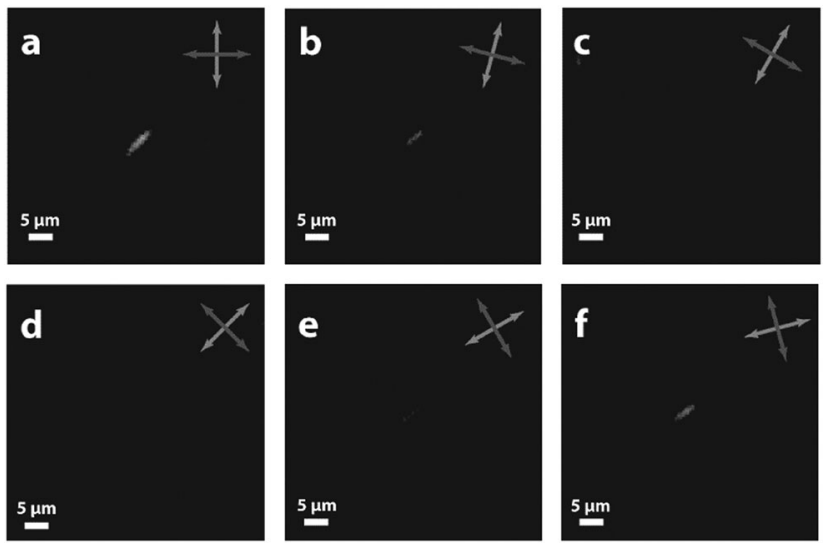

Fig. 2 Polarized optical microscopy images of a birefringent superstructure. The direction of the object relative to the crossed polarizers (represented by double arrows) was changed gradually by 15 degree increments.

The maximum extinction or maximum brightness should thus occur in a regular $45^{\circ}$ periodicity, which appears to be the case for the sample in Fig. 2. The birefringence of the PEDOT-S decorated superstructures gives a hint that the net orientation of the long axis of PEDOT-S is roughly parallel to the long axis of the superstructure.

Our investigation using POM microscopy made it plausible that PEDOT-S is decorating the fibril superstructure in an anisotropic fashion. The arrangement of PEDOT-S on the helical template can be further probed by Circular Dichroism spectroscopy (CD): if the polymer is arranged in a helical fashion onto the helical template this should be possible to analyse using CD. As birefringence is more of a qualitative technique, CD investigation would also allow us to examine the interaction in more detail. It should be possible to determine not only if the PEDOT-S can arrange itself in a chiral manner but also if the samples display right or left-handed chirality.

Many small molecules of a flat shape bind strongly to amyloid fibrils. $^{20,21}$ Of these, some linear dyes will bind to hydrophobic grooves formed due the presence of extensive beta-sheet arrays. In fact, this type of binding forms the basis for identification of amyloid by various optical probes such as Thioflavin T (ThT) ${ }^{20}$ and Congo Red (CR). ${ }^{22}$ The binding of such flat aromatic dyes to fibrils can lead to an alignment of the long axis of the dyes along the long fibril axis. ${ }^{23}$ It has been previously shown that binding of Congo Red to amyloid fibrils induces circular dichroism in the achiral dye. ${ }^{24}$ The mechanism of chiral transfer may be a twisting of the CR molecule upon binding to the amyloid structure or the arrangement of achiral CR molecules into a chiral helical pattern. ThT, in contrast to CR, does not show an induced CD-signal upon binding to amyloid fibrils. This allows ThT to be used as a probe for the formation of helical fibril superstructures. Upon addition of ThT to helical superstructures, ThT does display an induced CD-signal. ${ }^{14}$ During formation of the helical superstructures from bovine insulin, the system undergoes a bifurcation resulting in formation of an excess of superstructures showing an induced ThT signal that is either positive (labeled as +ICD) or negative (labeled as -ICD). Chiral amyloid superstructures can be a

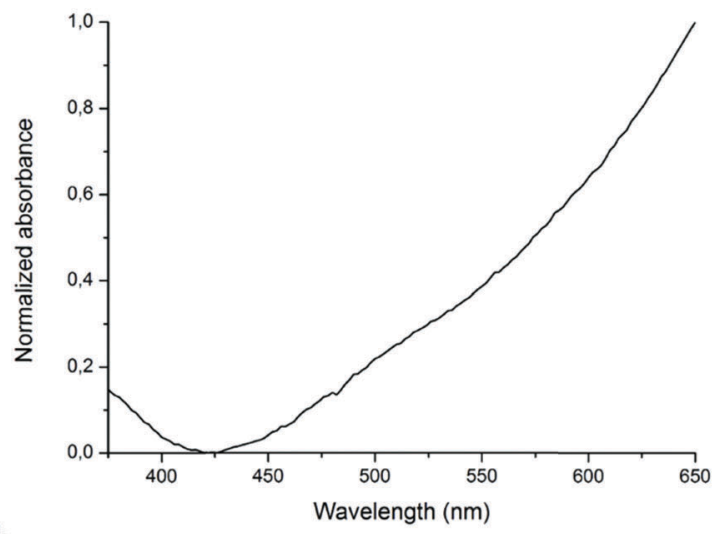

b

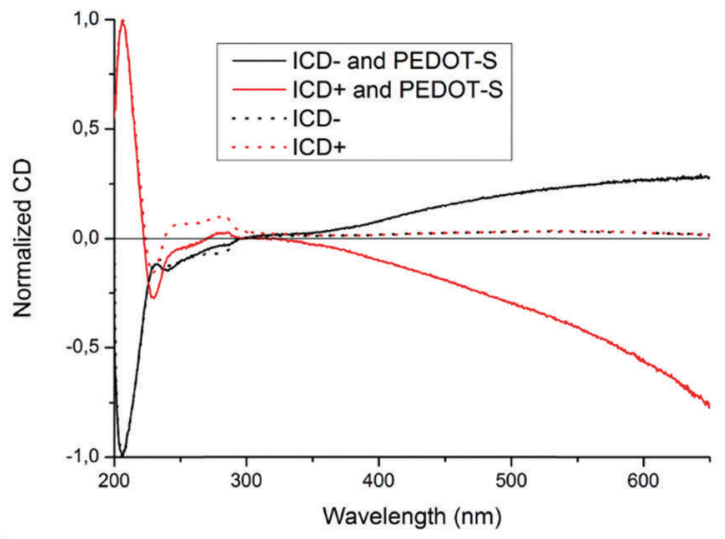

C

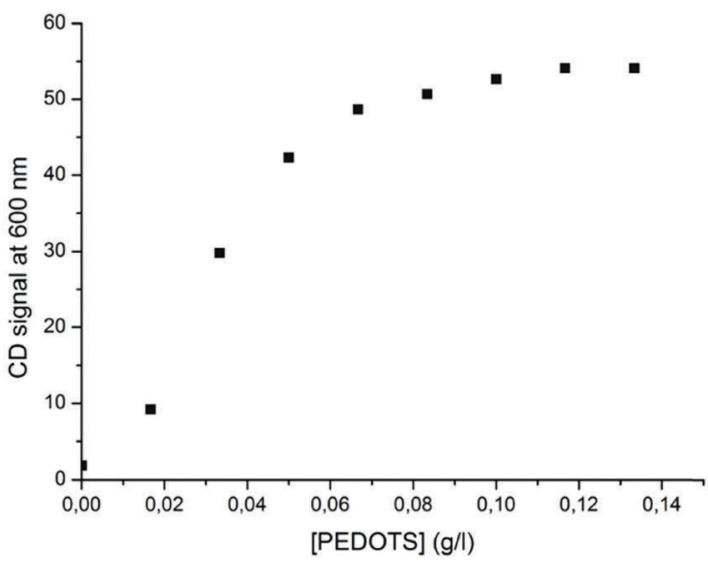

Fig. 3 (a) PEDOT-S absorption curve. (b) CD spectra of Insulin fibril superstructures (dashed) and PEDOT-S covered insulin fibril superstructures (solid lines) in $100 \mathrm{mM} \mathrm{NaCl}$. (c) $6.7 \mathrm{mg} \mathrm{l}^{-1}$ Insulin fibril superstructures with increasing concentration of PEDOT-S.

formed by agitation of acidic insulin solutions in the presence of $\mathrm{NaCl}$. Typical CD spectra of the resulting superstructures are shown in Fig. 3b for "right-handed" [+ICD] and "left-handed" [-ICD] structures. The chirality of these twisted superstructures is categorized according to the sign of induced circular dichroism for ThT-bound fibrils. ${ }^{14}$ PEDOT-S is mainly absorbing in the red part of the visible spectrum (for reference, the absorption spectrum of PEDOT-S shown in Fig. 3a) where no CD signal is present in the native superstructure sample. 
As PEDOT-S is added to the dispersion of superstructures an induced CD-signal (ICD-signal) appears (Fig. 3b) with a similar absorbance profile as that of PEDOT-S ( $c f$. Fig. 3a and b). The appearance of the ICD-signal indicates that PEDOT-S is organized by the helical protein superstructures. The ICD-signal of PEDOT-S is either positive or negative depending on the helicity (+ICD or -ICD) of the protein superstructures. However, at wavelengths below $400 \mathrm{~nm}$, the appearance of the CD-spectra obtained from PEDOT-S mixed with protein superstructures deviates from the PEDOT-S absorbance spectrum. This difference may be due to the protein-PEDOT-S complex containing multiple transition moments and thus causing the CD spectra to exhibit vertically inverted band signals compared to one or more of those seen in the corresponding spectra derived from regular absorbance measurements. In addition, CD signals originating from the protein will dominate at the lower wavelengths of the spectrum.

The presence of an induced CD-signal allows a more thorough investigation of the PEDOT-S:superstructure interaction. As only PEDOT-S bound to superstructures display an ICD signal, the ICD-signal gives an estimation of the amount of PEDOT-S bound to superstructures. Accordingly, we gradually added small amounts of PEDOT-S to a fixed amount of superstructures and measured the induced CD signal. The resulting graph (Fig. 3c) is typical of a saturation process where a sharp increase in the ICD signal with increased amount of PEDOT-S is followed by a more gradual increase towards a plateau. Interestingly, the level of bound PEDOT-S at saturation, approximate 1:1 PEDOT-S : protein $(\mathrm{w} / \mathrm{w})$ is similar to the experimental results where we added a large excess of PEDOT-S and washed away excess polymer. These result indicate a strong interaction between PEDOT-S and the superstructure, where the bound polymer is arranged in a chiral manner.

Having demonstrated the chiral organization of PEDOT-S by fibril superstructures in the liquid phase, we wanted to proceed to investigate the PEDOT-S:protein complexes in the solid state. Sample preparation is, however, complicated by the fact that low ionic strength and/or high $\mathrm{pH}$ will lead to disassembly of the helical structures. ${ }^{8}$ Thus the superstructures are formed as well as stored in acidified $100 \mathrm{mM} \mathrm{NaCl}$ solution. This presents a problem when attempting to deposit the complexes onto a surface since the high salt concentration will interfere with the adherence of the superstructures to the surface. Moreover, salt crystals, that are detrimental to any surface analysis technique, will form and cause light scattering. It is therefore desirable to dramatically decrease the salt concentration. However, it has been established that high ionic strength is required to preserve the helical superstructures. This has been demonstrated by observing a loss of the induced CD-signal from ThT as the salt concentration is decreased. ${ }^{14}$ We have therefore performed parallel investigations using SEM and CD that further reveals the effect of incubation in water on PEDOT-S functionalized protein superstructures. Samples were prepared where the protein superstructures were diluted with MilliQ water to a final $\mathrm{NaCl}$ concentration of $1 \mathrm{mM}$. In one type of sample the superstructures were diluted before addition of PEDOT-S. In the other type of sample, PEDOT-S was added prior to dilution.
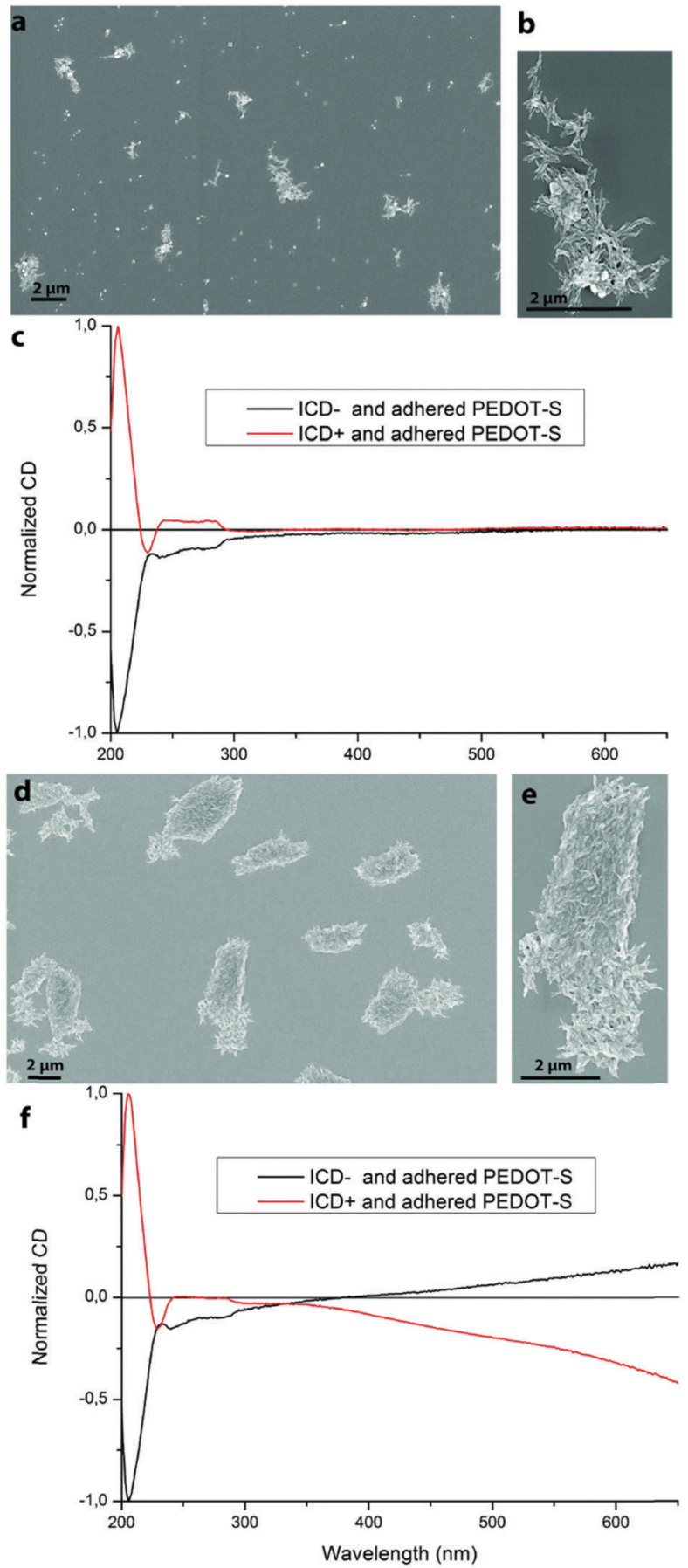

Fig. 4 Insulin fibril superstructures diluted in water. (a) SEM image of water incubated insulin fibril superstructures. (b) Close up of one of the structures in (a). (c). CD spectra of water incubated insulin fibril superstructures with subsequently added PEDOT-S. (d) SEM image of washed -ICD PEDOT-S-covered insulin fibril superstructures. (e) Close up of one of the structures in (d). (f) CD spectra of washed PEDOT-S-covered insulin fibril superstructures.

Interestingly, dramatic differences were obtained for these samples. SEM imaging of superstructures where PEDOT-S was added after the structures were diluted in MilliQ-water shows a surface littered with uneven and disrupted aggregates (Fig. 4a and b) In addition, a small amount of $\mathrm{NaCl}$ salt crystals can be 
observed. The same type of sample, where PEDOT-S was added after decreasing the salt concentration, investigated with CD did not show any discernable induced CD-signal (Fig. 4c). The ability of the superstructures to chirally organize PEDOT-S is thus lost under conditions of low ionic strength. In contrast, the addition of PEDOT-S to the protein superstructure prior to lowering of the ionic strength dramatically improves the stability of the entire complex. When such samples are investigated by SEM, images are obtained such as those shown in Fig. 4d and e where the PEDOT-S coated superstructures show no difference in morphology in comparison with typical protein superstructures. Moreover, the ability of the superstructures to chirally organize PEDOT-S is retained, as demonstrated by examination of such samples by CD (Fig. 4f), where a significant induced CD signal can be observed.

The retained CD signal and the SEM studies demonstrate that, unlike naked superstructures, which are highly sensitive towards a change in salt concentration, the PEDOT-S:fibril complex is stable towards dilution in water. The increased stability of PEDOT-S treated helical fibril assemblies is useful from a preparative standpoint and follows the findings by Gilbert et al. who used cat-ionic polymers to stabilize beta-lactoglobulin fibrils. ${ }^{25}$ Firstly, it allows for the deposition of helical fibrils onto surfaces while avoiding the presence of excess salt, thereby avoiding the formation of salt crystals. Secondly, since unbound PEDOT-S has been removed, the induced CD signal can only originate from PEDOT-S decorated superstructures. A sample of washed PEDOT-S decorated superstructures was drop-casted onto a glass surface and the CD signal was measured on the dry sample. As showed in ESI, $\uparrow$ Fig. S4 an induced CD signal is detected and thus the ability of protein template to organize PEDOT-S in a chiral fashion is retained in the solid state. As in the liquid phase, the ICD-signal of PEDOT-S changes from positive to negative depending on if it is associated with - ICD or +ICD helical superstructures. Measurements on solid state films with CD are complicated due to the presence of absorbance flattening and/or linear dichroism artefacts. Absorbance flattening would reduce the absorbance signal in high absorbance regions which would skew the appearance of the CD-signal causing e.g. an apparent red shift. ${ }^{26}$ Linear dichroism artifacts would affect the measured relation between right handed and left handed polarized light due to the right handed and/or the left handed light coming from the sample being contaminated by a portion of linearly polarized light. ${ }^{27}$ In of this study, however, neither of these two artifacts are likely explanations for the observed ICD-signal given that the recorded ICD signal changes sign for samples of PEDOT-S covered +ICD and -ICD helical superstructures. Although the appearance of the spectra of the solid state films may still be influenced by artifacts, the most likely explanation for the induced CD-signal is that PEDOT-S remains oriented in a helical fashion by the protein superstructures. We do note, as depicted in ESI, $\dagger$ Fig. S5, that in high resolution SEM the inherent helical structure of the superstructures is very difficult to discern. A possible explanation could be that the superstructures are much shorter than the full helix or that the helicity is of a length scale much smaller than the resolution of the SEM.

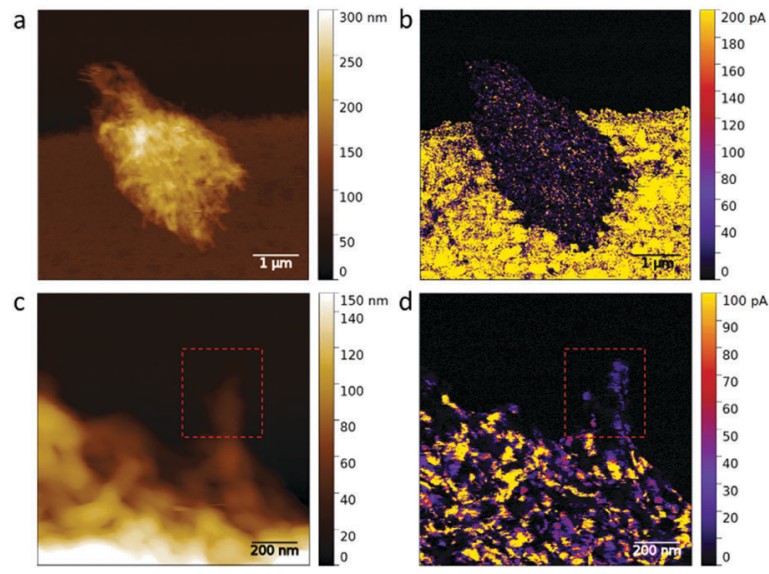

Fig. 5 Topography ( $a$ and $c$ ) and current maps ( $b$ and $d$ ) of fibril superstructures. Images in ( $a$ and b) show a structure at the edge of ITO/glass electrode. Images in (c and a) close-up of another superstructure where conducting small filaments are visible.

In addition to the chiral properties given to PEDOT-S by decoration of the protein superstructures, the ability of protein associated PEDOT-S to conduct current was investigated. In order to eliminate current originating from free PEDOT-S (not associated with the insulin superstructures) the PEDOT-S decorated superstructures was centrifuged and washed three times with MilliQ water, according to a previously developed procedure. ${ }^{12}$ A sample of the washed dispersion was drop cast onto ITO patterned glass substrates and the PEDOT-S:protein superstructures were analyzed using C-AFM. Fig. 5 shows current associated with the superstructures in contact with the ITO electrode, while no current was indeed detected on structures which were not in contact to the electrodes. In the larger magnification image (Fig. $5 \mathrm{c}$ and d) it is also possible to discern smaller filaments in the topography as well as in the corresponding current map (see the fibril structures highlighted by the red box), thus confirming the uniform attachment of conducting material to the amyloid insulin fibrils. The C-AFM current, measured at fixed voltage bias, is nearly independent of the tip-ITO distance, indicating a contact limited current. Indeed, due to the size of these structures and their wide cross section, the current is not limited by the material bulk resistance, but it is dominated by the injection at the tip-sample contact. ${ }^{28}$ In this framework the evaluation of the conductivity of the amyloid superstructures is not straightforward. However by comparing the average resistance measured for these structures (around $2 \mathrm{G} \Omega$ ) to that of PEDOT-S films under similar conditions ${ }^{29}$ (few $\mathrm{M} \Omega$ ) one could roughly estimate an out-of-plane conductivity of the decorated amyloid structures of around three orders of magnitude lower than the pure PEDOT-S films. The lower conductivity is more likely ascribed to the presence of insulating amyloid material within the interlaced structures. Nevertheless, due to the electrostatic nature of the amyloid fibers-PEDOT-S interactions, a partial de-doping effect, caused by the subtraction of sulphonate groups counterbalancing the positive charges on the PEDOT backbone, should also be considered. Due to the experimental limitations we are unable to discern any anisotropy in conductivity 
although previous results have shown an anisotropy alignment of polythiophenes onto amyloid fibrils. ${ }^{12,30}$

\section{Conclusions}

In conclusion, we have successfully used self-assembled chiral protein superstructures as a template to orient the achiral metallic polymer PEDOT-S into a helical form. By using either right handed or left handed protein superstructures as templates, a right handed or left handed helical orientation of PEDOT-S can be achieved. Moreover, the presence of PEDOT-S stabilizes the protein template at low ionic strength conditions. The resulting protein-PEDOT-S complex is stable towards dilution in water, and can be deposited onto surfaces, retaining the chirality. We have confirmed that the conductivity of PEDOT-S is retained and the conducting material appears to be ordered along the larger superstructures. The material components are prepared by self-assembly allowing for future modifications and fine tuning of properties. Future work will focus on tuning the superstructure assembly to create more uniform scaffolds and exploring surface depositioning techniques to enable Thz spectroscopy. The combination of helicity and conductivity is promising for applications involving interactions with electromagnetic waves.

\section{Acknowledgements}

Research has been funded by Swedish Government Strategic Research Area in Materials Science on Functional Materials at Linkoping University SFO-Mat-LiU 2009-00971, the Strategic Research Foundation through the project OPEN, and the Knut and Alice Wallenberg foundation with a Wallenberg Scholar grant to O.I.

\section{References}

1 S. Xu, Z. Yan, K.-I. Jang, W. Huang, H. Fu, J. Kim, Z. Wei, M. Flavin, J. McCracken, R. Wang, A. Badea, Y. Liu, D. Xiao, G. Zhou, J. Lee, H. U. Chung, H. Cheng, W. Ren, A. Banks, X. Li, U. Paik, R. G. Nuzzo, Y. Huang, Y. Zhang and J. A. Rogers, Science, 2015, 347, 154-159.

2 E. Yashima, K. Maeda, H. Iida, Y. Furusho and K. Nagai, Chem. Rev., 2009, 109, 6102-6211.

3 J. Chao, Y. Lin, H. Liu, L. Wang and C. Fan, Mater. Today, 2015, 18, 326-335.

4 K. Kamata, S. Suzuki, M. Ohtsuka, M. Nakagawa, T. Iyoda and A. Yamada, Adv. Mater., 2011, 23, 5509-5513.

5 K. Kamata, Z. Piao, S. Suzuki, T. Fujimori, W. Tajiri, K. Nagai, T. Iyoda, A. Yamada, T. Hayakawa, M. Ishiwara, S. Horaguchi, A. Belay, T. Tanaka, K. Takano and M. Hangyo, Sci. Rep., 2014, 4, 4919.
6 A. M. Kushner and Z. Guan, Angew. Chem., Int. Ed., 2011, 50, 9026-9057.

7 R. Khurana, C. Ionescu-Zanetti, M. Pope, J. Li, L. Nielson, M. Ramírez-Alvarado, L. Regan, A. L. Fink and S. A. Carter, Biophys. J., 2003, 85, 1135-1144.

8 L. Nielsen, S. Frokjaer, J. F. Carpenter and J. Brange, J. Pharm. Sci., 2001, 90, 29-37.

9 D. Ghosh, P. Dutta, C. Chakraborty, P. K. Singh, A. Anoop, N. N. Jha, R. S. Jacob, M. Mondal, S. Mankar, S. Das, S. Malik and S. K. Maji, Langmuir, 2014, 30, 3775-3786.

10 D. N. Woolfson and Z. N. Mahmoud, Chem. Soc. Rev., 2010, 39, 3464.

11 M. Hamedi, A. Herland, R. H. Karlsson and O. Inganäs, Nano Lett., 2008, 8, 1736-1740.

12 A. Elfwing, F. G. Bäcklund, C. Musumeci, O. Inganäs and N. Solin, J. Mater. Chem. C, 2015, 3, 6499-6504.

13 A. Loksztejn and W. Dzwolak, J. Mol. Biol., 2010, 395, 643-655.

14 A. Loksztejn and W. Dzwolak, J. Mol. Biol., 2008, 379, 9-16. 15 W. Dzwolak and M. Pecul, FEBS Lett., 2005, 579, 6601-6603.

16 M. Gruziel, W. Dzwolak and P. Szymczak, Soft Matter, 2013, 9, 8005.

17 K. M. Persson, R. Karlsson, K. Svennersten, S. Löffler, E. W. H. Jager, A. Richter-Dahlfors, P. Konradsson and M. Berggren, Adv. Mater., 2011, 23, 4403-4408.

18 R. H. Karlsson, A. Herland, M. Hamedi, J. A. Wigenius, A. Åslund, X. Liu, M. Fahlman, O. Inganäs and P. Konradsson, Chem. Mater., 2009, 21, 1815-1821.

19 A. O. Patil, Y. Ikenoue, N. Basescu, N. Colaneri, J. Chen, F. Wudl and A. J. Heeger, Synth. Met., 1987, 20, 151-159.

20 M. R. H. Krebs, E. H. C. Bromley and A. M. Donald, J. Struct. Biol., 2005, 149, 30-37.

21 K. P. R. Nilsson, FEBS Lett., 2009, 583, 2593-2599.

22 H. Puchtler, F. Sweat and M. Levine, J. Histochem. Cytochem., 1962, 10, 355-364.

23 F. G. Bäcklund, J. Wigenius, F. Westerlund, O. Inganäs and N. Solin, J. Mater. Chem. C, 2014, 2, 7811.

24 R. Khurana, V. N. Uversky, L. Nielsen and A. L. Fink, J. Biol. Chem., 2001, 276, 22715-22721.

25 J. Gilbert, O. Campanella and O. G. Jones, Biomacromolecules, 2014, 15, 3119-3127.

26 E. Castiglioni, S. Abbate, G. Longhi and R. Gangemi, Chirality, 2007, 19, 491-496.

27 B. Norden, A. Rodger and T. Dafforn, Linear Dichroism and Circular Dichroism, The Royal Society of Chemistry, London, 2010.

28 D. Moerman, N. Sebaihi, S. E. Kaviyil, P. Leclère, R. Lazzaroni and O. Douhéret, Nanoscale, 2014, 6, 10596.

29 W. Cai, C. Musumeci, F. N. Ajjan, Q. Bao, Z. Ma, Z. Tang and O. Inganäs, J. Mater. Chem. A, 2016, 4, 15670-15675.

30 A. Herland, P. Björk, P. R. Hania, I. G. Scheblykin and O. Inganäs, Small, 2007, 3, 318-325. 